\title{
Novel PNKP mutations causing defective DNA strand break repair and PARP1 hyperactivity in MCSZ
}

Ilona Kalasova, PhD, * Hana Hanzlikova, PhD, * Neerja Gupta, MD, DM, Yun Li, MD, Janine Altmüller, MD, John J. Reynolds, PhD, Grant S. Stewart, PhD, Bernd Wollnik, MD, Gökhan Yigit, PhD, and

Keith W. Caldecott, PhD

Neurol Genet 2019;5:e320. doi:10.1212/NXG.0000000000000320

\section{Abstract}

\section{Objective}

To address the relationship between novel mutations in polynucleotide 5'-kinase 3'-phosphatase (PNKP), DNA strand break repair, and neurologic disease.

\section{Methods}

We have employed whole-exome sequencing, Sanger sequencing, and molecular/cellular biology.

\section{Results}

We describe here a patient with microcephaly with early onset seizures (MCSZ) from the Indian sub-continent harboring 2 novel mutations in PNKP, including a pathogenic mutation in the fork-head associated domain. In addition, we confirm that MCSZ is associated with hyperactivation of the single-strand break sensor protein protein poly (ADP-ribose) polymerase 1 (PARP1) following the induction of abortive topoisomerase I activity, a source of DNA strand breakage associated previously with neurologic disease.

\section{Conclusions}

These data expand the spectrum of PNKP mutations associated with MCSZ and show that PARP1 hyperactivation at unrepaired topoisomerase-induced DNA breaks is a molecular feature of this disease.

\author{
Correspondence \\ Dr. Hanzlikova \\ hana.hanzlikova@ \\ img.cas.cz, or Dr. Gupta \\ neerja17aiims@gmail.com, or \\ Dr. Caldecott \\ k.w.caldecott@sussex.ac.uk
}

*These authors contributed equally.

From the Department of Genome Dynamics (I.K., H.H., K.W.C.), Institute of Molecular Genetics of the Czech Academy of Sciences, Czech Republic; Genome Damage and Stability Centre (H.H., K.W.C.), School of Life Sciences, University of Sussex, Falmer, Brighton, UK; Institute of Human Genetics (Y.L., B.W., G.Y.), University Medical Center Göttingen, Germany; Cologne Center for Genomics (J.A.), University of Cologne, Germany; Institute of Cancer and Genomic Sciences (J.J.R., G.S.S.), College of Medical and Dental Sciences, University of Birmingham, UK; and Division of Genetics (N.G.), Department of Pediatrics, All India Institute of Medical Sciences, New Delhi, India.

Funding information and disclosures are provided at the end of the article. Full disclosure form information provided by the authors is available with the full text of this article at Neurology.org/NG.

The Article Processing Charge was funded by the ERC.

This is an open access article distributed under the terms of the Creative Commons Attribution License 4.0 (CC BY), which permits unrestricted use, distribution, and reproduction in any medium, provided the original work is properly cited. 


\section{Glossary}

CCG $=$ Cologne Center for Genomics; CPT = camptothecin; FHA = fork-head associated; HRP $=$ horseradish peroxidase; $\mathbf{M C S Z}=$ microcephaly with early onset seizures; PARP1 = protein poly (ADP-ribose) polymerase $1 ; \mathbf{P N K P}=$ polynucleotide $5^{\prime}$ kinase 3'-phosphatase; SCAN-1 = spinocerebellar ataxia with axonal neuropathy-1; SSBR = single-strand break repair; WES = whole-exome sequencing.

Mutations in DNA single-strand break repair (SSBR) proteins are associated with hereditary neurologic disease. ${ }^{1,2}$ Recently, using a mouse model in which the SSBR protein $\mathrm{Xrccl}$ is mutated, we demonstrated that hyperactivation of the SSB sensor protein poly(ADPribose) polymerase 1 (PARP1) is a likely source of the neuropathology induced by SSBs. ${ }^{3}$ During SSBR, the enzyme polynucleotide $5^{\prime}$-kinase $3^{\prime}$-phosphatase (PNKP) can employ either DNA 3'-phosphatase and/or DNA 5' kinase activities to restore ligatable termini at DNA strand breaks, and is recruited to SSBs by interacting with XRCC1 via an amino-terminal fork-head associated (FHA) domain. ${ }^{4-6}$ Strikingly, PNKP mutations are associated with 2 apparently distinct neurologic diseases: microcephaly with early onset seizures (MCSZ) and ataxia with oculomotor apraxia $4 .^{7,8}$ The former is a neurodevelopmental disease associated with developmental delay, microcephaly, and seizures whereas the latter is a degenerative disease associated with cerebellar atrophy, ataxia, and oculomotor apraxia. Intriguingly, some individuals with PNKP mutations exhibit phenotypic aspects of both disorders, and in some cases can overlap with Charcot-Marie-Tooth disease. ${ }^{9,10}$ To explain the molecular basis and phenotypic diversity of PNKP-associated disease will require an understanding of the spectrum of PNKP mutations associated with disease, and of the influence of these mutations on DNA repair. Here, we identify 2 novel disease mutations in PNKP in a patient with MCSZ from the Indian subcontinent. We identify a defect in DNA strand break repair in patient-derived primary fibroblasts and that these MCSZ cells are associated with hyperactivation of the SSB sensor protein, PARP1.

Figure 1 Novel PNKP mutations in an individual from India with MCSZ

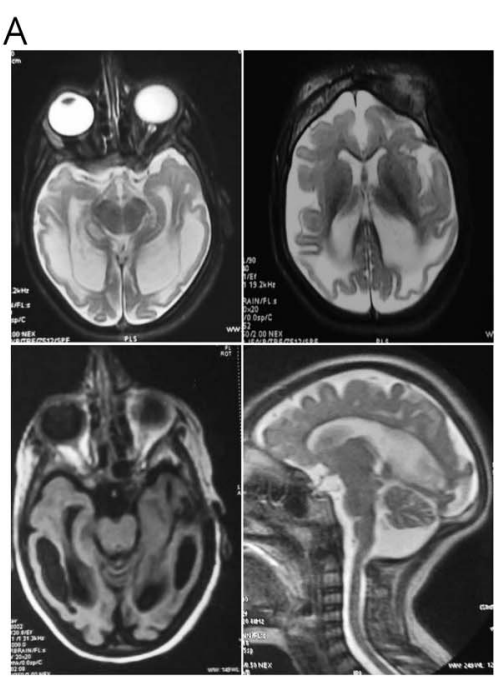

C

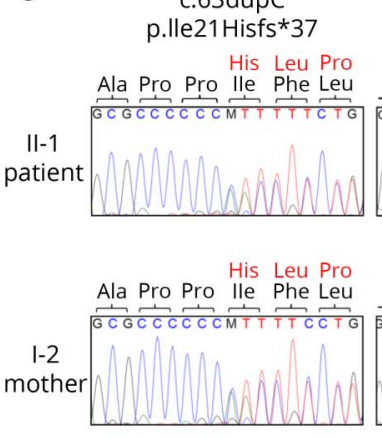

C. $1295 \_1298+6$ del

c. $12951298+6$ delCCAGGTAGCG $\frac{\text { Exon } 14}{\text { GCCGCGYSASS KMGCGKGMC }}$
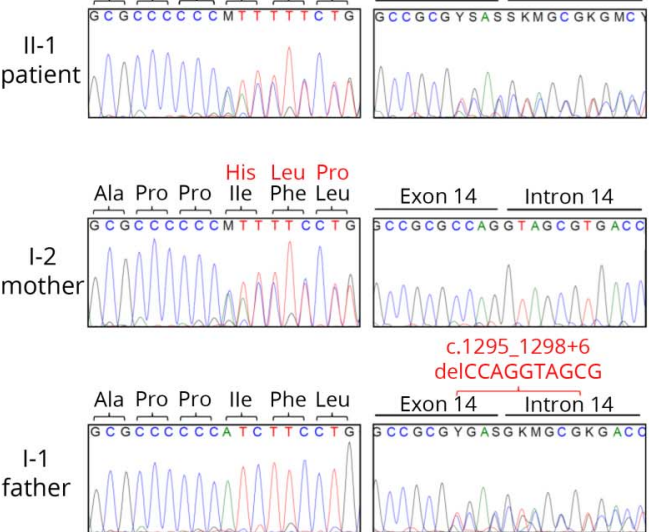

c.1295_1298+6

Exon 14

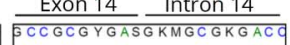

B

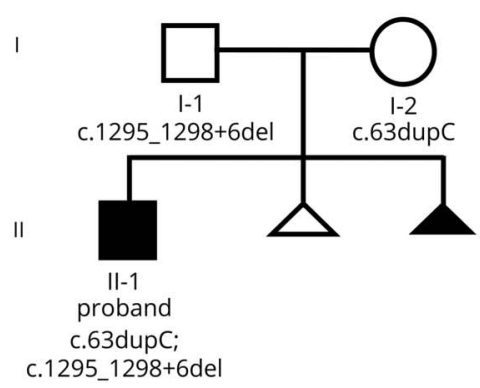

D

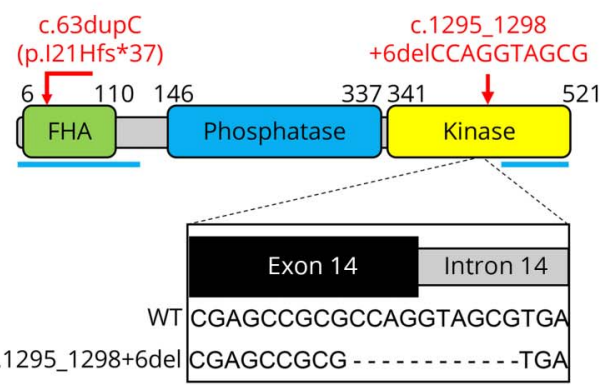

(A), patient MRI. T2 axial and FLAIR axial images showing microcephaly with diffuse white matter signal changes. There is mild ventriculomegaly with generalized atrophy and cystic changes in bilateral temporal lobes. There is mild hypoplasia of inferior vermis as seen on sagittal T2 weighted image. (B), Pedigree of the proband (black square). The triangles indicate pregnancies that did not go to term. Note that one of these (black triangle) was associated with antenatal detection of microcephaly. (C), Electropherograms showing the compound heterozygous PNKP mutations in the proband and heterozygosity in the parents. (D), cartoon of PNKP and its functional domains. The proband mutations, confirmed by Sanger sequencing, are shown in red. Epitopes of the $\mathrm{N}$ - and C-terminal polyclonal antibodies used in this study are indicated with horizontal blue lines. The inset shows the impact of the deletion caused by c.1295 $1298+6 \mathrm{del}$ on the splice site between exon 14 and intron 14. FLAIR = fluid-attenuated inversion recovery; MCSZ = microcephaly with early onset seizures; PNKP = polynucleotide 5' kinase 3'-phosphatase. 
A

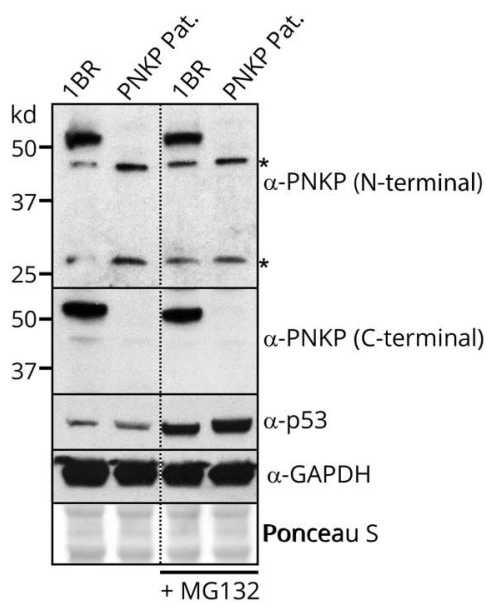

C
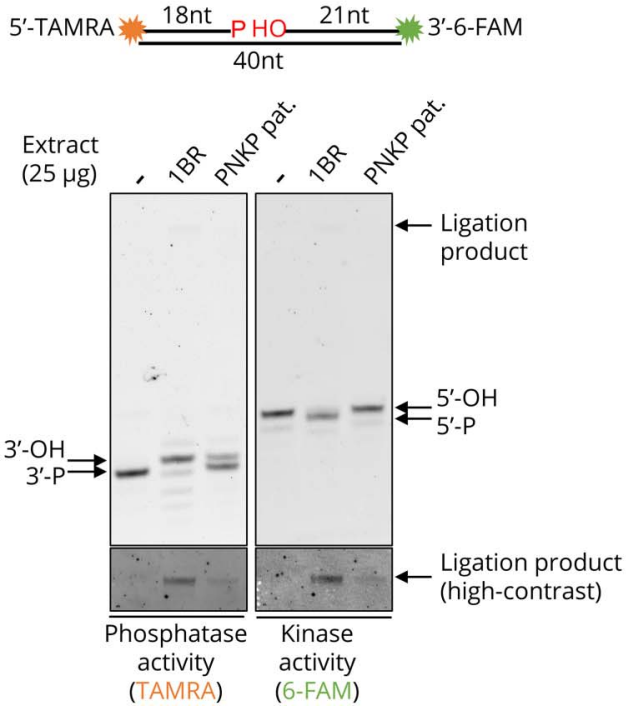

B

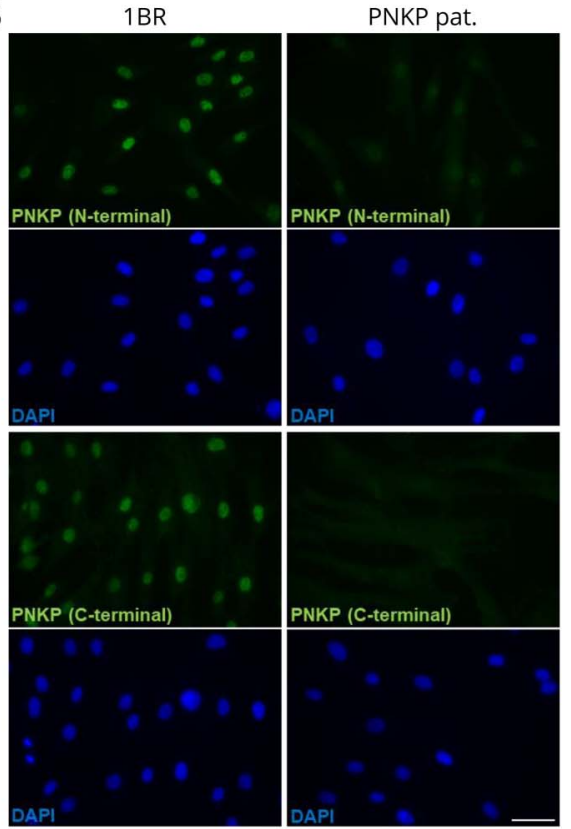

D

(A), western blot showing PNKP protein levels in 1BR and PNKP patient fibroblasts after 6 hours incubation with DMSO vehicle or $10 \mu \mathrm{M}$ proteasome inhibitor MG132. Two different antibodies were employed, directed at the $\mathrm{N}$ - or C-terminus as indicated. p53 and GAPDH detection was employed as a positive control for proteasome inhibition by MG132 and as a loading control, respectively. Asterisks denote nonspecific bands. (B), PNKP indirect immunofluorescence with the antibodies described above in 1BR and PNKP patient fibroblasts. Scale bar $50 \mu \mathrm{m}$ (C), PNKP DNA 3' -phosphatase and DNA 5'-kinase activity in wild type 1BR and PNKP patient cell extracts. Top, A cartoon of the PNKP oligonucleotide duplex substrate, harboring a SSB with 3'-phosphate and 5 '-hydroxyl termini. Bottom; reaction products of PNKP activity. The positions of the substrate ( $\left.3^{\prime}-\mathrm{P}\right)$ and product $\left(3^{\prime}-\mathrm{OH}\right)$ of PNKP $3^{\prime}$-phosphatase activity (left panel), and of the substrate $\left(5^{\prime}-\mathrm{OH}\right)$ and product (5'-P) of the $5^{\prime}$-kinase activity (right panel) are shown. The 40-mer ligation product of the repair reaction, which is labeled with both TAMRA and FAM, is indicated by an arrow in the top panel and is shown in the bottom panels with high contrast (D), PNKP DNA 3'-phosphatase activity in cell extracts from 1BR and PNKP patient fibroblasts additionally transfected with PNKP siRNA. The 40-mer ligation products are highlighted in the middle (high contrast) panel. The PNKP protein levels in the siRNA transfected 1BR and patient fibroblasts are shown at the bottom. MCSZ = microcephaly with early onset seizures; PNKP = polynucleotide 5'-kinase 3 '-phosphatase; TAMRA = tetramethylrhodamine.

\section{Methods}

\section{Patient case report}

The patient is currently a 8.5 -year-old boy who was born at term with microcephaly (occipito-frontal circumference 29 $\mathrm{cm},-3 \mathrm{SD})$ and below-normal weight (-2.8 SD). Seizures began at day 15 of his life and he had severe developmental delay. He established head control at 6 months and started sitting with support at month 7 , but never attained walking. He developed monosyllable speech at month 8 but since then has exhibited a complete developmental arrest. Examination at $\sim$ year 8.5 revealed severe microcephaly $(-14.9 \mathrm{SD})$, short stature $(-6.86 \mathrm{SD})$, and failure to thrive (weight $=-5.83 \mathrm{SD})$. $\mathrm{He}$ is a thin child with a slender build, convergent squint, receding forehead, high nasal bridge, bilateral up slant, receding chin, dental crowding and teeth caries, spasticity, brisk reflexes, and striatal toe. Ataxia could not be ascertained. He has a café-au-lait spot on his right upper thigh and dark hyperpigmented skin. He also has a large amplitude stereotype lower limb movements and midline hand stereotypes. Hearing evaluation revealed an absence of waves at $50 \mathrm{~dB}$ in the left ear. At year 4.5, T2 axial and fluid-attenuated inversion recovery axial MRI images revealed microcephaly with diffuse white matter signal changes (figure 1A). There was mild ventriculomegaly with generalized atrophy and cystic changes in bilateral temporal lobes. Mild hypoplasia of the inferior vermis was evident on the sagittal T2 weighted image. His CT scan brain did not show any calcifications. 
A

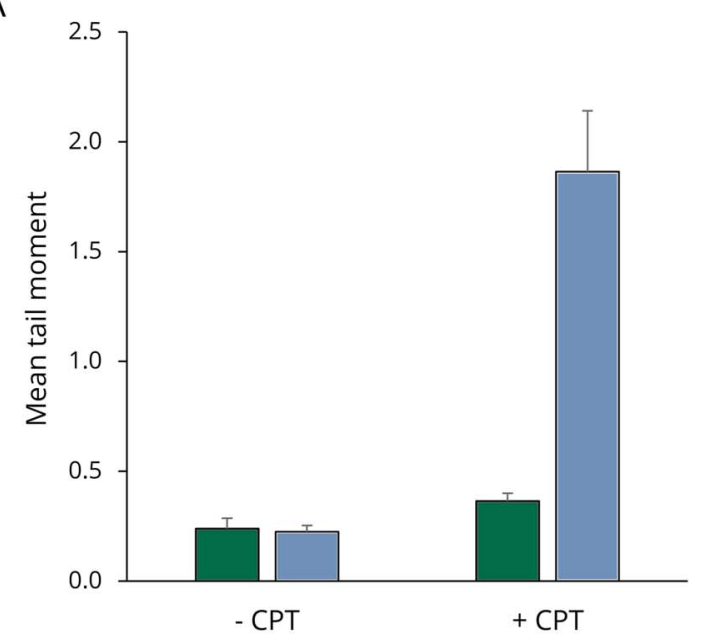

$\square 1 \mathrm{BR}$

$\square$ PNKP Patient

B
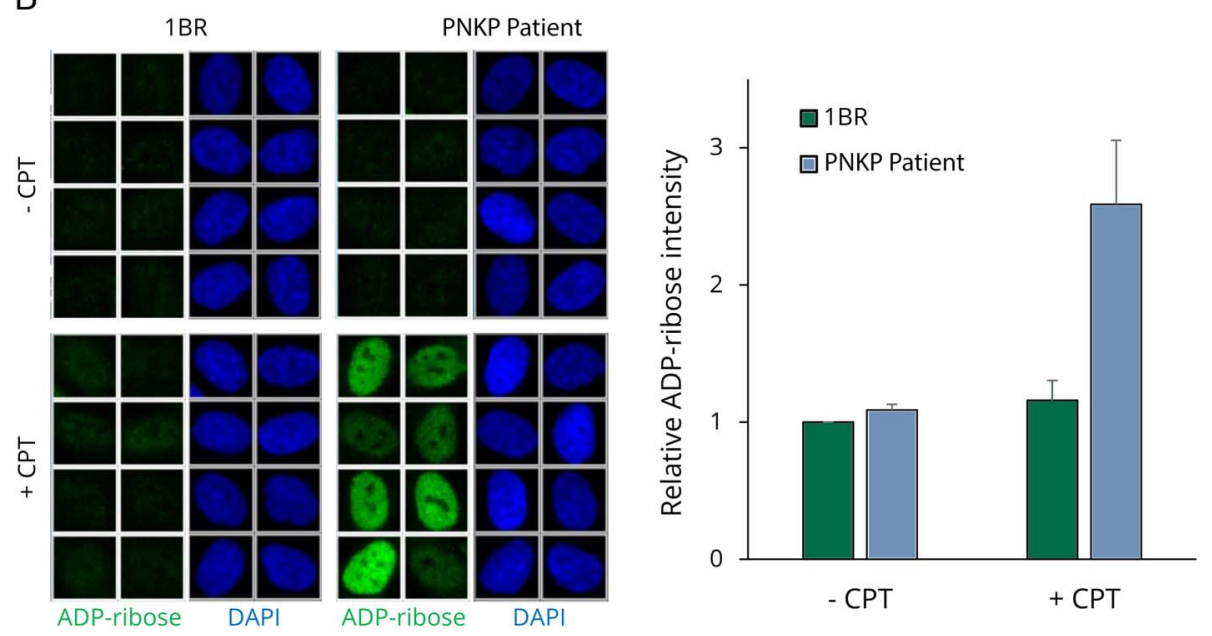

(A) The accumulation of CPT-induced DNA strand breaks in 1BR and patient fibroblasts, quantified by alkaline comet assays. Cells were treated with DMSO vehicle or $10 \mu \mathrm{M}$ CPT for 45 minutes. Data are the average comet tail moment of 100 cells per sample and are the mean ( \pm SEM) of 4 independent experiments. (B) Representative ScanR images (left) and quantitation (right) of poly (ADP-ribose) levels in control 1BR and PNKP patient fibroblasts incubated in the presence or absence of CPT as above. Data are the mean $( \pm$ SEM) of 3 independent experiments. $\mathrm{CPT}=$ camptothecin; $\mathrm{DMSO}=$ dimethyl sulphoxide; MCSZ = microcephaly with early onset seizures; PNKP = polynucleotide $5^{\prime \prime}$-kinase 3 "-phosphatase; SEM = standard error of the mean.

EEG at 5 years revealed resolving hypsarryhthmia and evolving multifocal epilepsy. His karyotype, liver function, and renal function were all normal. His serum uric acid was $1.2 \mathrm{mg} / \mathrm{dL}$. His serum a fetoprotein levels are $22.9 \mathrm{ng} / \mathrm{mL}$ (normal range $0.89-8.78 \mathrm{ng} / \mathrm{mL}$ ). His lipid profile showed mildly high triglycerides 161 (normal range 50-150) with low density lipoprotein/high density lipoprotein ratio 3.44. $\mathrm{He}$ is at present non-ambulatory, can sit with support, and does not have seizures.

\section{Whole exome sequencing}

Whole-exome sequencing (WES) was performed on DNA extracted from blood lymphocytes of the index patient using the SureSelectXT Human All Exon V6 enrichment kit (Agilent technologies) on an Illumina HiSeq4000 sequencer. In total, we obtained a mean coverage of 84 reads, and $96.3 \%$ of target were covered more than 10x. WES data analysis and variant filtering was conducted using the exome analysis pipeline "varbank" of the Cologne Center for Genomics (CCG, varbank.ccg.uni-koeln.de/) and data were filtered for high-quality (coverage of more than 6 reads, a minimum quality score of 10 ), rare (minor allele frequency $<0.5 \%$ in the databases of the exome aggregation consortium and single nucleotide polymorphisms) autosomal recessive variants.

\section{Mutation screening}

Variants identified by WES were amplified from DNA of the index patients, and its parents and PCR products were sequenced by BigDye Terminator method on an ABI $3500 \times$ L Genetic Analyzer (Life Technologies, Germany). Identified mutations were re-sequenced in independent experiments.

\section{Primary human fibroblasts}

Control human fibroblast 1BR3 (denoted 1BR in the text) and PNKP patient-derived skin fibroblasts, obtained with appropriate patient consent, were grown in minimum essential media (Gibco) supplemented with $15 \%$ fetal bovine serum, $2 \mathrm{mM}$ glutamine, the antibiotics penicillin (100 units/ $\mathrm{mL})$, and streptomycin $(100 \mu \mathrm{g} / \mathrm{mL})$ in a humidified atmosphere of $5 \% \mathrm{CO}_{2}$ at low oxygen $(5 \%)$ at $37^{\circ} \mathrm{C}$. 


\section{Antibodies, western blotting, and indirect immunofluorescence microscopy}

Primary antibodies were anti-pan-ADP-ribose binding reagent (MABE1016, Millipore), anti-PNKP FHA (SK3195), anti-PNKP C-terminal (ab18107, Abcam), anti-p53 (OP29, Millipore), and anti-GAPDH (sc-47724, Santa Cruz). Secondary antibodies were horseradish peroxidase (HRP) conjugated goat anti-rabbit (170-6515, Bio-Rad) and goat anti-mouse (170-6516, Bio-Rad) and for indirect immunofluorescence donkey anti-rabbit Alexa 488 (A21206, Invitrogen). For western blotting, cells were lysed in sodium dodecyl sulphate (SDS) sample buffer and subjected to SDSPAGE, transferred onto nitrocellulose membrane and detected by specific antibodies combined with HRP conjugated secondary antibodies. For indirect immunofluorescence microscopy, cells were cultured on glass coverslips and treated where indicated with $10 \mu \mathrm{M}$ camptothecin (CPT) (Sigma) for 45 minutes. Cells were fixed with $4 \%$ paraformaldehyde and immunostained as described previously. ${ }^{3}$ Images were taken using a DMi6000 microscope (Leica) with 40x dry objective. Automated wide-field microscopy was performed on ScanR system (Olympus) with ScanR Image Acquisition and Analysis Software, 40x/0.95NA (UPLSAPO 2 40X) dry objective.

\section{PNKP biochemical activity assays}

PNKP substrate was prepared by annealing equimolar amounts of the fluorophore-labeled deoxyriboligonucleotides (Midland Certified Reagent Company) "S1" [5'-(TAMRA)-TAGCATCGATCAGTCCTC-3'-P], "S2” [5'-OH-GAGGTCTAGCATCGTTAGTCA-(6-FAM)-3'] and a complementary strand oligonucleotide [5'-TGACTAACGATGCTAGACCTCTGAGGACTGATCGATGCTA-3'] in annealing buffer (10 mM Tris pH 7.5, $200 \mathrm{mM} \mathrm{NaCl}, 1 \mathrm{mM}$ EDTA). Cell-free protein extracts were prepared in lysis buffer $[25 \mathrm{mM}$ Tris, pH 7.5, 10 mM EDTA, 10 mM EGTA, $100 \mathrm{mM} \mathrm{NaCl}, 1 \%$ Triton X-100, cOmplete protease inhibitors (Roche)] and incubated $(25 \mu \mathrm{g}$ total protein) with $50 \mathrm{nM}$ substrate and $1 \mu \mathrm{M}$ single-stranded nuclease competitor oligonucleotide $\left[5^{\prime}\right.$ AAAGATCACAAGCATAAAGAGACAGG-3'] in reaction buffer (25 mM Tris, $\mathrm{pH} 7.5,130 \mathrm{mM} \mathrm{KCl}, 10 \mathrm{mM} \mathrm{MgCl}$, $1 \mathrm{mM} \mathrm{DTT}, 1 \mathrm{mM} \mathrm{ATP}$ ) at $37^{\circ} \mathrm{C}$ for 10 minutes in $50 \mu \mathrm{L}$ reactions and were terminated by addition of $50 \mu \mathrm{L}$ quenching buffer (90\% formamide, 50 mM EDTA, 0.006\% Orange G). 10 $\mu \mathrm{L}$ was separated on $20 \%$ denaturing polyacrylamide gels and analyzed on a PharosFX Molecular Imager System (Bio-Rad). Where indicated, cells were transfected with mix of either PNKP siRNA \# 1: 5' -CCGGAUAUGUCCACGUGAA-3' and PNKP siRNA \#2: 5'-GGAAACGGGUCGCCAUCGA-3' or non-target siRNA \#1: 5' -UGGUUUACAUGUCGACUAA-3' and non-target siRNA \#2: 5'-UGGUUUACAUGUUGUGUGA-3') using Lipofectamine RNAiMAX (Life Technologies) 72 hours before lysis.

\section{Cellular DNA strand break assays}

The steady-state level of DNA strand breaks was evaluated by alkaline comet assays essentially as described ${ }^{11}$ following mock-treatment or treatment with $10 \mu \mathrm{M} \mathrm{CPT}$ for 60 minutes at $37^{\circ} \mathrm{C}$. The average comet tail moment in 100 cells per sample was evaluated by Comet Assay IV software (Perceptive Instruments).

\section{Standard protocol approvals, registrations, and patient consents}

Ethical approval and informed parental consent for the use and publication of patient information and patient derived cell lines was obtained from the appropriate Institutional and regional committees. No vertebrate animal models were employed in this work.

\section{Data availability}

Data will be provided on request at https://sussex.figshare. com/10.25377/sussex.7836653.

\section{Results}

Individuals with neurologic disease resulting from mutations in PNKP protein have been identified in the Americas, Europe, Middle East, and Japan, but surprisingly not yet on the Indian subcontinent. Here, we describe the first Indian patient with biallelic mutations in PNKP. The proband is an 8.5 year old boy with microcephaly, early-onset seizures, and developmental delay (MCSZ). Seizures were detected from day 15 of life and MRI revealed microcephaly with diffuse white matter signal changes and generalized atrophy in the bilateral temporal lobes (figure 1A). A detailed case report is presented in the Methods.

WES on DNA extracted from blood lymphocytes from the proband identified 2 heterozygous variants in PNKP (NM 007254.3); c.63dupC and c.1295_1298+6delCCAGGTAGC $\bar{G}$ (figure $1 \mathrm{~B}$ and C). Sanger sequencing confirmed the presence of both mutations in the proband and their heterozygosity in the parents. c.63dupC is a 1 base pair duplication in exon 2 and was inherited from the mother, and c.1295_1298+6delCCAGGTAGCG is a 10 base pair deletion that was inherited from the father (figure $1 \mathrm{~B}$ and $\mathrm{C}$ ). The 1 base pair duplication is predicted to cause a frameshift resulting in nonsense-mediated mRNA decay and/or truncated PNKP protein (p.lle21Hisfs*37) and is the first unambiguously deleterious mutation identified in the FHA domain (figure 1D). The 10 base pair deletion spans the last 4 nucleotides of exon 14 and the first 6 nucleotides of intron 14 (figure 1C) and deletes a donor splice-site, leading most likely to nonsense-mediated mRNA decay and/or a synthesis of a truncated protein.

To define the consequence of the 2 novel PNKP mutations at the molecular and cellular level, we employed primary fibroblasts established from a skin biopsy from the proband. Western blotting using antibodies targeting either the $\mathrm{N}$-terminal or C-terminal regions of PNKP failed to detect PNKP protein in the patient-derived fibroblasts, even if we incubated the cells with the proteasome inhibitor MG132 to 
prevent the degradation of the mutant PNKP (figure 2A). Incubation with MG132 increased the level of p53 protein, however, confirming that the proteasome was inhibited in these experiments. The impact of the 2 novel mutations on PNKP protein levels was also observed by indirect immunofluorescence, with both N-terminal and C-terminal PNKP antibodies again detecting little or no PNKP in the patientderived fibroblasts (figure $2 \mathrm{~B}$ ).

Next, we measured the level of PNKP activity using a sensitive biochemical assay. ${ }^{12}$ This assay uses an oligonucleotide duplex substrate possessing an internal single-nucleotide gap with $3^{\prime}$-phosphate and $5^{\prime}$-hydroxyl termini, which is labeled at the ends of the duplex with the fluorescent dyes tetramethylrhodamine and 6-carboxyfluorescein (figure 2C). The 3'phosphate and $5^{\prime}$-hydroxyl termini in this substrate were completely repaired by incubation with cell extract from wild type fibroblasts within 10 minutes. Although greatly reduced, DNA $3^{\prime}$-phosphatase and $5^{\prime}$-kinase activities were also detected in the patient cell extract as indicated by the formation of a small amount of fully repaired reaction product ("ligation product") and also in the case of 3 '-phosphatase activity by the appearance of a small amount of dephosphorylated oligonucleotide (figure 2C left panel,“ 3 ' $\mathrm{OH}$ ). This residual activity in the patient-derived fibroblasts was greatly reduced or ablated if the patient cells were first transfected with PNKP siRNA, indicating that it reflected residual levels of PNKP (figure 2D). It is currently unclear how the residual PNKP activity is generated in the patient cells, especially with respect to $5^{\prime}$-kinase activity, because both mutations are predicted to result in translational frameshifts and to truncate either almost all of the protein (in the case of the maternal allele, c.63dupC) or a large region of the kinase domain (in the case of the paternal allele, c.1295_1298+6del) (figure 1D). Possible explanations include a low level of alternative splicing, an alternative translation start site downstream of the premature stop codon in the maternal PNKP allele, a small amount of properly spliced mRNA arising from the paternal allele, or the presence of a small amount of an alternative $5^{\prime}$-kinase.

We next examined whether the greatly reduced level of PNKP protein and activity in the patient cells affected the rate of DNA strand break repair. DNA strand breaks induced by abortive topoisomerase 1 (TOP1) activity possess termini that are substrates for both the DNA kinase and DNA phosphatase activities of PNKP. Indeed, the PNKP patient fibroblasts accumulated higher levels of DNA strand breaks than 1BR control cells during incubation with TOP1 'poison' CPT (figure 3A). This observation is significant, because defects in the repair of TOP1-induced DNA breaks are also observed in cells from other MCSZ patients and from patients with spinocerebellar ataxia with axonal neuropathy-1 and ataxia telangiectasia, illustrating their relevance to neurodegenerative disease $^{13-15}$. Importantly, hyperactivation of the SSB sensor protein PARP1 is observed in and is causally linked with SSBR-defective cerebellar ataxia. ${ }^{3}$ Consequently, we wondered whether PARP1 hyperactivation might also be a feature of these MCSZ cells. Indeed, strikingly, PNKP patient fibroblasts accumulated much higher levels of the poly (ADP-ribose) product of PARP1 activity than did 1BR control fibroblasts during treatment with CPT (figure 3B).

Our data identify 2 novel PNKP mutations in a patient with MCSZ from India. Of interest this patient exhibits one of the most severe forms of MCSZ documented, with a level of microcephaly and growth retardation comparable to patients with ataxia telangiectasia and $\operatorname{Rad} 3$ related-Seckel Syndrome. ${ }^{16}$ Despite this, in keeping with the functional analysis of other identified PNKP mutations, ${ }^{15}$ cells derived from this patient retain some residual PNKP activity. This is consistent with the observation that complete deletion of PNKP in mouse is embryonic lethal. ${ }^{17}$ Finally, we have confirmed that PARP1 hyperactivation is a cellular hallmark of MCSZ cells, highlighting this phenomenon in the development of a range of clinically distinct neuro-pathologies.

\section{Author contributions}

I. Kalasova: designed and conducted the experiments. $\mathrm{H}$. Hanzlikova: designed, conducted, and coordinated the experiments. N. Gupta: identified and characterized the patient and initiated the study. J. Altmüller: conducted WES. Y. $\mathrm{Li}$ : analyzed the WES Data and identified the mutations. J. Reynolds: initiated and consulted on the study. G. Stewart: initiated and consulted on the study. B. Wollnik: supervised the WES. G. Yigit: supervised the WES. K.W Caldecott: managed and coordinated the study.

\section{Acknowledgment}

This work was funded by an ERC advanced investigator award to KWC (SIDSCA; 694996). Access to the Olympus ScanR and Leica microscope at the Light Microscopy Core Facility, IMG CAS, Prague was supported by MEYS (LM2015062), OPPK (CZ.2.16/3.1.00/21547), and NPU the authors (LO1419). This work used instruments provided by C4Sys infrastructure. GSS is funded by a CR-UK programme grant (C17183/A23303) and JJR is funded by the University of Birmingham.

\section{Study funding}

The study was funded by an MRC Programme grant to KWC (MR/P010121/1) and an ERC Advanced Investigator Award to KWC (SIDSCA; 694996).

\section{Disclosure}

I. Kalasova, H. Hanzlikova, and N. Gupta report no disclosures. J. Altmüller has nothing to disclose. Y. Li reports no disclosures. J. Reynolds has received academic research support from the University of Birmingham and has received foundation/society support from Cancer Research UK. Grant Stewart has served on the Editorial Board of Oncogene and Molecular and Cellular Biology and has received foundation/ society support from Cancer Research UK. B. Wollnik and G. 
Yigit report no disclosures. Keith Caldecott has served on the Editorial Board for DNA Repair and has received government research support from the Medical Research Council, Cancer Research UK, and the European Research Council. Full disclosure form information provided by the authors is available with the full text of this article at Neurology.org/NG.

\section{Publication history}

Received by Neurology: Genetics November 6, 2018. Accepted in final form February 7, 2019.

\section{References}

1. Jiang B, Glover JNM, Weinfeld M. Neurological disorders associated with DNA strand-break processing enzymes. Mech Ageing Dev 2017;161(pt A):130-140.

2. Yoon G, Caldecott KW. Nonsyndromic cerebellar ataxias associated with disorders of DNA single-strand break repair. Handb Clin Neurol 2018;155:105-115.

3. Hoch NC, Hanzlikova H, Rulten SL, et al. XRCC1 mutation is associated with PARP1 hyperactivation and cerebellar ataxia. Nature 2016;541:87-91.

4. Jilani A, Ramotar D, Slack C, et al. Molecular cloning of the human gene, PNKP, encoding a polynucleotide kinase 3'-phosphatase and evidence for its role in repair of DNA strand breaks caused by oxidative damage. J Biol Chem 1999;274: 24176-24186.

5. Karimi-Busheri F, Daly G, Robins P, et al. Molecular characterization of a human DNA kinase. J Biol Chem 1999;274:24187-24194.

6. Loizou JI, El-Khamisy SF, Zlatanou A, et al. The protein kinase CK2 facilitates repair of chromosomal DNA single-strand breaks. Cell 2004;117:17-28.
7. Shen J, Gilmore EC, Marshall CA, et al. Mutations in PNKP cause microcephaly, seizures and defects in DNA repair. Nat Genet 2010;42:245-249.

8. Bras J, Alonso I, Barbot C, et al. Mutations in PNKP cause recessive ataxia with oculomotor apraxia type 4. Am J Hum Genet 2015;96:474-479.

9. Leal A, Bogantes-Ledezma S, Ekici AB, et al. The polynucleotide kinase 3'-phosphatase gene (PNKP) is involved in Charcot-Marie-Tooth disease (CMT2B2) previously related to MED25. Neurogenetics 2018;19:215-225.

10. Pedroso JL, Rocha CRR, Macedo-Souza LI, et al. Mutation in PNKP presenting initially as axonal Charcot-Marie-Tooth disease. Neurol Genet 2015;1:e30.

11. Breslin C, Clements PM, El-Khamisy SF, Petermann E, Iles N, Caldecott KW. Measurement of chromosomal DNA single-strand breaks and replication fork progression rates. Meth Enzymol 2006;409:410-425.

12. Dobson CJ, Allinson SL. The phosphatase activity of mammalian polynucleotide kinase takes precedence over its kinase activity in repair of single strand breaks. Nucleic Acids Res 2006;34:2230-2237.

13. El-Khamisy SF, Saifi GM, Weinfeld M, et al. Defective DNA single-strand break repair in spinocerebellar ataxia with axonal neuropathy-1. Nature 2005;434:108-113.

14. Katyal S, Lee Y, Nitiss KC, et al. Aberrant topoisomerase-1 DNA lesions are pathogenic in neurodegenerative genome instability syndromes. Nat Neurosci 2014;17: 813-821.

15. Reynolds JJ, Walker AK, Gilmore EC, Walsh CA, Caldecott KW. Impact of PNKP mutations associated with microcephaly, seizures and developmental delay on enzyme activity and DNA strand break repair. Nucleic Acids Res 2012;40: 6608-6619.

16. O'Driscoll M, Ruiz-Perez VL, Woods CG, Jeggo PA, Goodship JA. A splicing mutation affecting expression of ataxia-telangiectasia and Rad3-related protein (ATR) results in Seckel syndrome. Nat Genet 2003;33:497-501.

17. Shimada M, Dumitrache LC, Russell HR, McKinnon PJ. Polynucleotide kinasephosphatase enables neurogenesis via multiple DNA repair pathways to maintain genome stability. EMBO J 2015;34:2465-2480. 


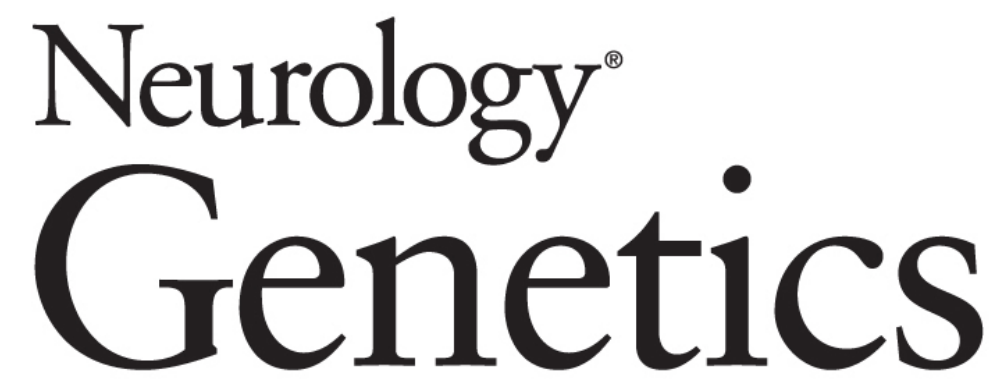

Novel PNKP mutations causing defective DNA strand break repair and PARP1 hyperactivity in MCSZ

Ilona Kalasova, Hana Hanzlikova, Neerja Gupta, et al. Neurol Genet 2019;5;

DOI 10.1212/NXG.0000000000000320

This information is current as of March 25, 2019

Neurol Genet is an official journal of the American Academy of Neurology. Published since April 2015, it is an open-access, online-only, continuous publication journal. Copyright Copyright () 2019 The Author(s). Published by Wolters Kluwer Health, Inc. on behalf of the American Academy of Neurology.. All rights reserved. Online ISSN: 2376-7839.

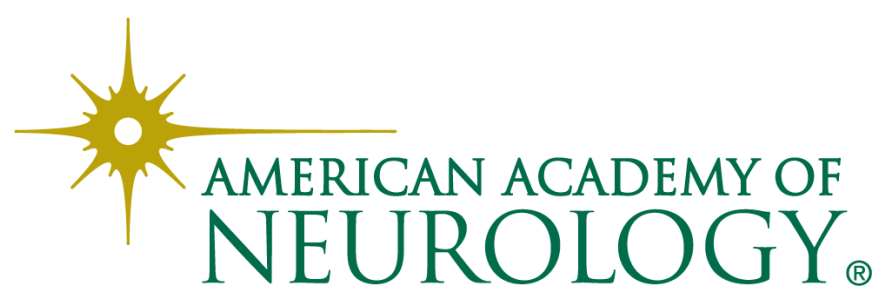




\section{Updated Information \& Services}

References

Citations

Subspecialty Collections

Permissions \& Licensing

Reprints including high resolution figures, can be found at: http://ng.neurology.org/content/5/2/e320.full.html

This article cites 17 articles, 4 of which you can access for free at: http://ng.neurology.org/content/5/2/e320.full.html\#\#ref-list-1

This article has been cited by 6 HighWire-hosted articles: http://ng.neurology.org/content/5/2/e320.full.html\#\#otherarticles

This article, along with others on similar topics, appears in the following collection(s):

All Epilepsy/Seizures

http://ng.neurology.org//cgi/collection/all_epilepsy_seizures All Genetics http://ng.neurology.org//cgi/collection/all_genetics

\section{All Movement Disorders}

http://ng.neurology.org//cgi/collection/all_movement_disorders

Information about reproducing this article in parts (figures,tables) or in its entirety can be found online at:

http://ng.neurology.org/misc/about.xhtml\#permissions

Information about ordering reprints can be found online:

http://ng.neurology.org/misc/addir.xhtml\#reprintsus

Neurol Genet is an official journal of the American Academy of Neurology. Published since April 2015, it is an open-access, online-only, continuous publication journal. Copyright Copyright $\odot 2019$ The Author(s). Published by Wolters Kluwer Health, Inc. on behalf of the American Academy of Neurology.. All rights reserved. Online ISSN: 2376-7839.

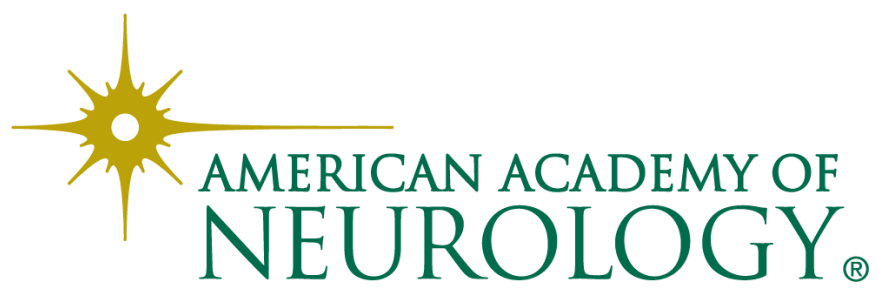

